\title{
Infrared study of Large scale h-BN film and Graphene/h-BN heterostructure
}

\author{
Kwangnam Yu, ${ }^{1}$ Jiho Kim, ${ }^{1}$ Chul Lee, ${ }^{1}$ A-Rang Jang, ${ }^{2}$ Hyeon \\ Suk Shin, ${ }^{2}$ Keun Soo Kim, ${ }^{3}$ Young-Jun Yu, ${ }^{4}$ and E. J. Choi ${ }^{1, *}$ \\ ${ }^{1}$ Department of Physics, University of Seoul, Seoul 130-743, Republic of Korea \\ ${ }^{2}$ Department of Chemistry, Ulsan National Institute of Science and Technology (UNIST), Ulsan 689-798, Republic of Korea \\ ${ }^{3}$ Department of Physics and Graphene Research Institute, \\ Sejong University, Seoul 143-747, Republic of Korea \\ ${ }^{4}$ Creative Research Center for Graphene Electronics, \\ Electronics and Telecommunications Research Institute (ETRI), Daejeon 305-700, Korea
}

(Dated: November 11, 2019)

\begin{abstract}
We synthesize a series of CVD h-BN films and perform critical infrared spectroscopic characterization. For high-temperature $\left(\mathrm{HT}\right.$, Temp $\left.=1400{ }^{\circ} \mathrm{C}\right)$ grown h-BN thin film only $E_{1 \mathrm{u}}$-mode infrared phonon is activated demonstrating highly aligned $2 \mathrm{D}$ h-BN planes over large area, whereas low-temperature $\left(\mathrm{LT}, \mathrm{Temp}=1000{ }^{\circ} \mathrm{C}\right.$ ) grown film shows two phonon peaks, $E_{1 \mathrm{u}}$ and $A_{2 \mathrm{u}}$, due to stacking of h-BN plane at tilted angle. For CVD graphene transferred on $\mathrm{HT}$ h- $\mathrm{BN} / \mathrm{SiO}_{2} / \mathrm{Si}$ substrate, interband transition spectrum $\sigma_{1}$ shifts strongly to lower energy compared with that on LT h-BN $/ \mathrm{SiO}_{2} / \mathrm{Si}$ and on bare $\mathrm{SiO}_{2} / \mathrm{Si}$ substrate, revealing that residual carrier density $n$ in graphene is suppressed by use of HT h-BN layer. Also the interband transition width of $\sigma_{1}$ defined by effective temperature is reduced from $400 \mathrm{~K}$ for $\mathrm{G} / \mathrm{SiO}_{2} / \mathrm{Si}$ to $300 \mathrm{~K}$ for $\mathrm{HT}$ h-BN/SiO $2 / \mathrm{Si}$. The behaviors of $n$ and effective temperature show that HT h-BN film can decouple CVD graphene from the impurity and defect of $\mathrm{SiO}_{2}$ leading to large scale free-standing like graphene.
\end{abstract}

\section{INTRODUCTION}

Hexagonal boron nitride (h-BN) is a graphite-like layered material characterized by wide band gap $\sim 6 \mathrm{eV}$. 1] The $\mathrm{B}$ and $\mathrm{N}$ atoms bond covalently to constitute BN honeycomb planes, whereas adjacent $\mathrm{BN}$ planes are weakly coupled by van der Waals interaction. 2] h-BN shows a range of attractive properties such as hightemperature stability, high mechanical strength, and large thermal conductivity useful for application. [ $[-3]$ In particular when used as substrate for graphene h$\mathrm{BN}$ greatly enhances carrier mobility at room- and low-temperature compared with conventional $\mathrm{SiO}_{2} / \mathrm{Si}$ substrate. [7-9] The superior performance as substrate is directly associated with the ultraflat, chemically inert surface and also the high purity, defect-free bulk nature of h-BN. [10, 11] However, the exfoliated h-BN flake is limited in size by small lateral length, only $\sim 10 \mu m \times 10 \mu m$, which seriously hinders its use in application. For practical industrial application large scale $\mathrm{h}-\mathrm{BN}$ is strongly needed.

Recently large area h-BN thin films are synthesized by CVD technique. 12 16] Here chemical precursors containing $\mathrm{B}$ and $\mathrm{N}$ are thermally evaporated and deposited on metallic platforms $(\mathrm{Cu}, \mathrm{Pt}, \mathrm{Fe})[12-15]$ or $\alpha-\mathrm{Al}_{2} \mathrm{O}_{3}$ crystal. [16] Hexagonal phase BN film is formed for certain ranges of vapor pressure and growth temperature. The h-BN film is isolated from its template and transfered onto desired substrate such as $\mathrm{SiO}_{2} / \mathrm{Si}$. Further transfer of a pregrown CVD graphene sheet on h-BN/substrate completes the large scale fabrication of graphene sup-

\footnotetext{
* Corresponding author: echoi@uos.ac.kr
}

ported by h-BN buffer layer. 17] A systematic spectroscopic study and critical comparison with the exfoliated samples, currently lacking, can lead to deeper understanding of the large area samples.

In this work we perform infrared transmission measurement on the CVD h-BN and graphene/h-BN films. For the h-BN film infrared-active phonon peaks are probed. A correlation of the phonon profile with the growth condition is found, providing a knowledge about the lattice structure of the films. For graphene/h-BN interband transition of Dirac carrier in graphene is measured using the infrared spectroscopy. From the electronic transition we are able to determine Fermi energy, carrier density, and defect-related interband transition width of graphene.

\section{EXPERIMENT}

We grew multilayer h-BN thin films using CVD method on $\mathrm{Al}_{2} \mathrm{O}_{3}$ substrate at two different temperatures (Temp) $1000{ }^{\circ} \mathrm{C}$ and $1400{ }^{\circ} \mathrm{C}$. The details of the film synthesis were reported elsewhere. [18] The h-BN films were isolated from the $\mathrm{Al}_{2} \mathrm{O}_{3}$ and transferred on IRtransparent $\mathrm{SiO}_{2} / \mathrm{p}$-Si substrate, $1 \mathrm{~cm} \times 1 \mathrm{~cm}$ in size, by using electrochemical bubbling-based method. [14, 18] We also prepared single crystal multilayer h-BN flakes by exfoliation. Large scale monolayer graphene was grown on Cu-foil (Alfa Aesar, 99.999\%) using CVD method. 19, 20] The CVD graphene was then transfered on the CVD h$\mathrm{BN} / \mathrm{SiO}_{2} / \mathrm{p}$-Si following the PMMA transfer method. 17] Infrared transmission was measured using a microscope FTIR spectrometer (Bruker Vertex 70V). Optical dielectric functions were calculated by fitting transmission and reflection data using RefFit analysis program. 21] 

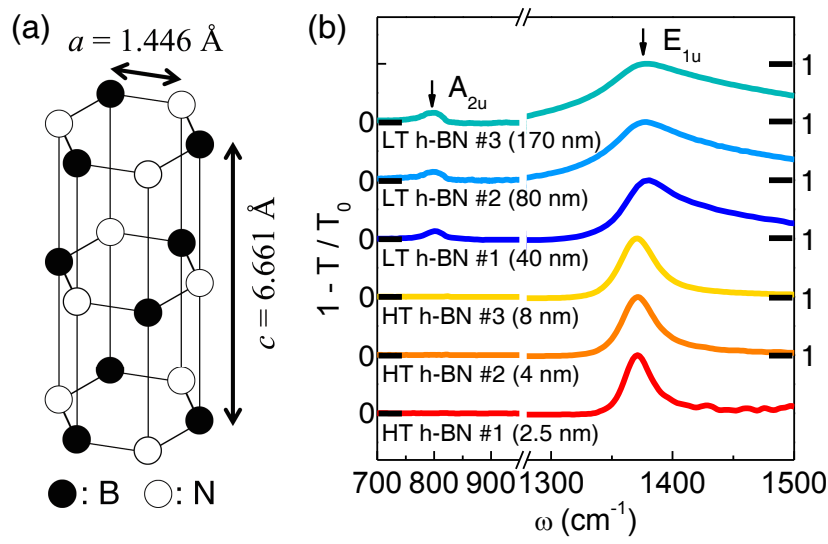

FIG. 1.

(a) Lattice structure of h-BN: space group $=\mathrm{P} 6_{3} / \mathrm{mmc}$, lattice constants $a=1.446 \AA, c=$ $6.661 \AA$. 3] (b) $1-T / T_{0}$ of h-BN film grown at lowtemperature $\left(\mathrm{LT}\right.$, Temp $=1000{ }^{\circ} \mathrm{C}$ ) and high-temperature $\left(\mathrm{HT}\right.$, Temp $\left.=1400^{\circ} \mathrm{C}\right) . T$ and $T_{0}$ stand for infrared transmission of h-BN/substrate and bare substrate respectively. For LT h-BN, $A_{2 \mathrm{u}}$ phonon is observed at $\omega=800 \mathrm{~cm}^{-1}$ which is absent for the HT h-BN film. For each spectrum the intensity is normalized by that of $E_{1 u}$ peak. The numbers in the parenthesis show the thicknesses of the h-BN films.

\section{RESULT}

\section{A. h-BN thin film}

Figure 1 (b) shows $1-T / T_{0}$ of the h-BN films grown at low-temperature $\left(\mathrm{LT}\right.$, Temp $\left.=1000{ }^{\circ} \mathrm{C}\right)$ and hightemperature $\left(\mathrm{HT}\right.$, Temp $\left.=1400{ }^{\circ} \mathrm{C}\right)$. For LT h-BN two IR-active phonon peaks are observed at $\omega=800 \mathrm{~cm}^{-1}$ and $\omega=1375 \mathrm{~cm}^{-1}$. On the other hand, for HT h$\mathrm{BN}$, the low-frequency peak is absent and only the highfrequency $E_{1 \mathrm{u}}$ peak is activated. The $E_{1 \mathrm{u}}$ phonon represents in-plane oscillation of $\mathrm{B}$ and $\mathrm{N}$ atoms within the BN plane. The $\omega=800 \mathrm{~cm}^{-1}$ phonon is considered to be a $c$-axis mode associated with vibration normal to the BN plane. 22, 23] For our near-normal incident experiment, infrared light should couple only with the in-plane $E_{1 \mathrm{u}}$ phonon mode if the BN-plane of the thin film were perfectly aligned with the substrate-plane. The phonon profiles of LT h-BN and HT h-BN suggest that the two kinds of films differ in their lattice alignments.

To better understand the phonon behavior we performed angle-tilted IR transmission measurement using single crystal h-BN. Here $100 \mathrm{~nm}$-thick h-BN crystal is tilted from $\theta=0$ to $30^{\circ}$ with respect to the incident IR as sketched in Figure 2(a). As $\theta$ increase $E_{1 \mathrm{u}}$ peak intensity decrease and that of $A_{2 \mathrm{u}}$ peak increases. The peak intensity $I\left(A_{2 \mathrm{u}}\right)$ and $I\left(E_{1 \mathrm{u}}\right)$ change with $\theta$ as $I\left(A_{2 \mathrm{u}}\right) \sim \sin \theta$ and $I\left(E_{1 \mathrm{u}}\right) \sim \cos \theta$ (data not shown). The $\theta$-dependent behaviors of the IR phonons confirm that they are inplane and out-of-plane vibration modes respectively. In Figure 2(b) we plot the intensity ratio $I\left(A_{2 \mathrm{u}}\right) / I\left(E_{1 \mathrm{u}}\right)$ as function of $\theta$. The ratio increases in proportion to $\tan \theta$,
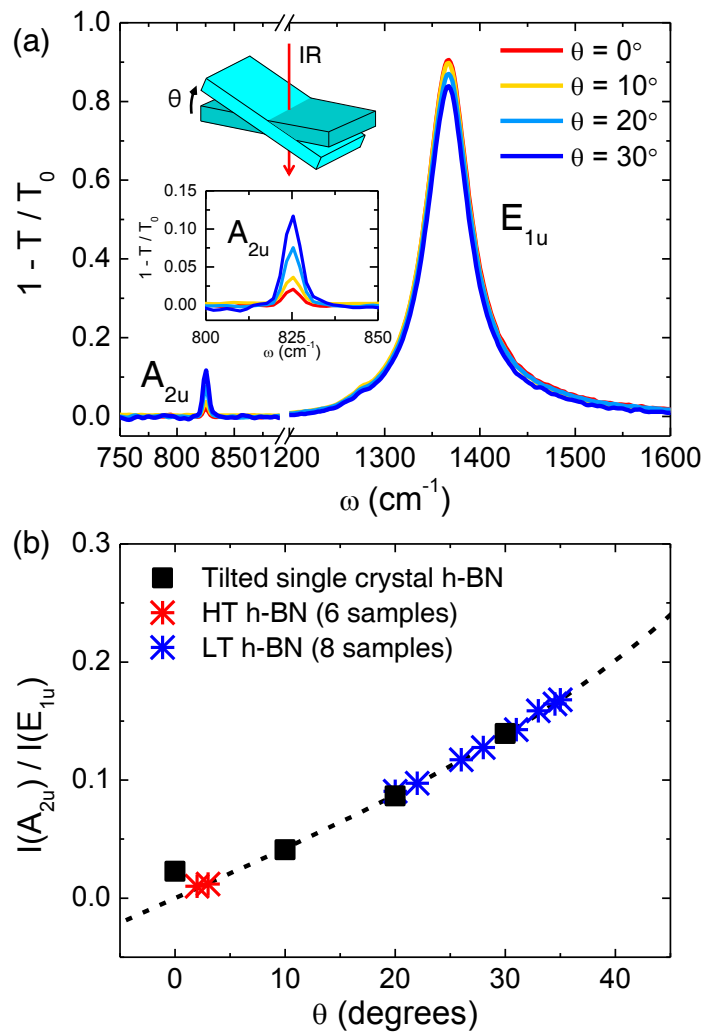

FIG. 2. (a) $1-T / T_{0}$ measured for a single crystal h-BN flake. As the tilting angle $\theta$ increases, the $A_{2 u}$ phonon intensity increases (see the inset) and $I\left(E_{1 \mathrm{u}}\right)$ phonon intensity decreases respectively. (b) Ratio of peak heights $I\left(A_{2 \mathrm{u}}\right)$ and $I\left(E_{1 \mathrm{u}}\right)$ as function of $\theta$. The dashed curve, $0.24 \tan \theta$, connects the single crystal data points. Based on this curve we infer the tilting angle of LT h-BN films $\theta=20^{\circ} \sim 40^{\circ}$ from their phonon intensity ratio $0.1<I\left(A_{2 \mathrm{u}}\right) / I\left(E_{1 \mathrm{u}}\right)<0.15$. For $\mathrm{HT}$ h-BN films we have $I\left(A_{2 \mathrm{u}}\right) / I\left(E_{1 \mathrm{u}}\right)<0.01$ and $\theta<3^{\circ}$.

$I\left(A_{2 \mathrm{u}}\right) / I\left(E_{1 \mathrm{u}}\right)=A \tan \theta(A=0.24)$, as shown by the dashed curve. We observe very weak, non-zero $A_{2 \mathrm{u}}$ peak at $\theta=0^{\circ}$ because in our microscope spectrometer the IR light is focused on the sample with near-normal incidence angle. For LT h-BN the phonon intensity of the samples, eight in total, is confined in $0.1<I\left(A_{2 \mathrm{u}}\right) / I\left(E_{1 \mathrm{u}}\right)<0.15$. In terms of $\theta$ it corresponds to the tilting of the $\mathrm{BN}$ plane by $\theta=20^{\circ} \sim 40^{\circ}$ according to the $I\left(A_{2 \mathrm{u}}\right) / I\left(E_{1 \mathrm{u}}\right)$ curve map. In contrast all HT h-BN samples, six in total, show $I\left(A_{2 \mathrm{u}}\right) / I\left(E_{1 \mathrm{u}}\right)<0.01$ and therefore small tilting of the BN plane, $\theta<3^{\circ}$. For the film synthesis at Temp $=1000{ }^{\circ} \mathrm{C}(\mathrm{LT})$ our result shows that although the $\mathrm{B}$ and $\mathrm{N}$ precursors do form the hexagonal $\mathrm{BN}$ phase in CVD chamber, the $\mathrm{BN}$ planes fail to conform to the [0001] plane of the $\mathrm{Al}_{2} \mathrm{O}_{3}$ substrate. Instead tilted growth of $\mathrm{BN}$ plane is energetically favored. For hightemperature Temp $=1400{ }^{\circ} \mathrm{C}$ growth the tilting problem is largely cured, leading to almost perfectly aligned BN planes over the entire area of the large h-BN/substrate sample.

High quality film growth often becomes challenging in 

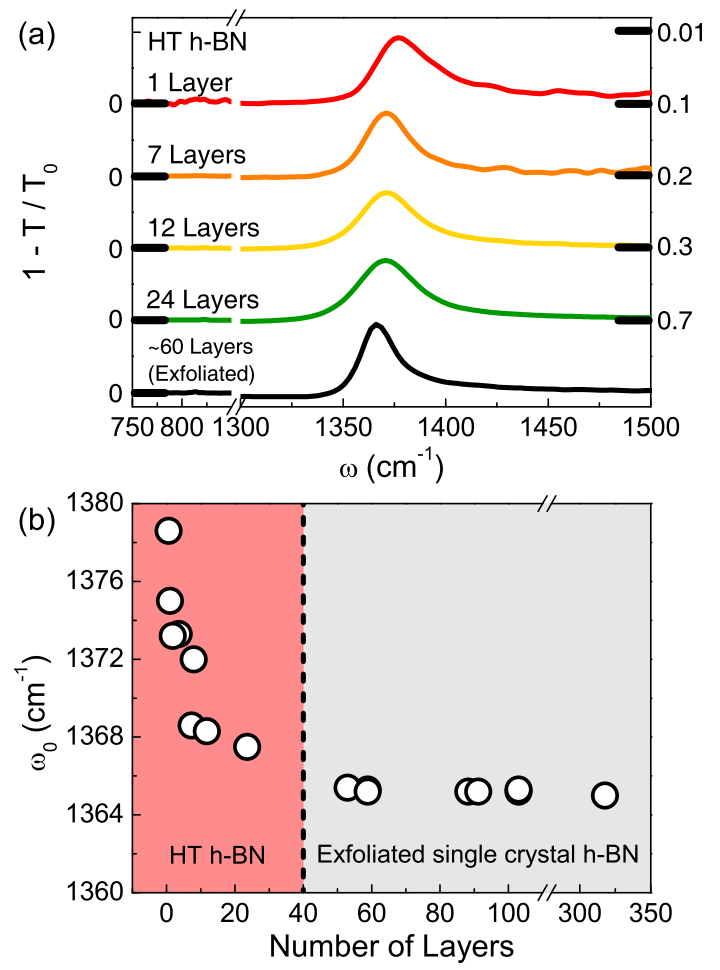

FIG. 3. (a) Infrared phonon of HT h-BN with the film thickness ranging from 24 layers to 1 layer. For comparison, the data for thick single crystal h-BN flake is shown together. The curves are displaced vertically for clarity. (b) $E_{1 \mathrm{u}}$ phonon frequency $\omega_{0}$ against the h-BN thickness for the HT h-BN films $(1 \sim 40$ layers $)$ and single crystal h-BN flakes $(>40$ layers).

the ultrathin, few-layer thickness region. To investigate how the $E_{1 \mathrm{u}}$ phonon change with thickness, we measured IR transmission for a series of $\mathrm{HT}$ h-BN samples with controlled thickness. Figure $3(\mathrm{a})$ shows $1-T / T_{0}$ of HT h-BN from $d=24$ layers to $d=1$ layer. We used the relation $0.33 \mathrm{~nm}=1$ layer to convert the unit of thickness from nm to the number of layers. The characteristic phonon spectrum, prominent $E_{1 \mathrm{u}}$ peak and absence of $A_{2 \mathrm{u}}$ peak, persists for all samples, indicating the aligned growth of h-BN layer at few layer and monolayer film. Interestingly the $E_{1 \mathrm{u}}$ peak shifts to higher energy as the film becomes thinner. In Figure $3(\mathrm{~b})$ we plot the phonon frequency $\left(\omega_{0}\right)$ of h-BN film as function of h-BN thickness. $\omega_{0}$ measured for several exfoliated h-BN crystal flakes are shown together. For thick h-BN flakes $(d>40$ layers) $\omega_{0}$ is independent with $d$ and it agrees with the frequency of bulk h-BN crystal. For HT h-BN films $\omega_{0}$ increases rapidly as $d$ decreases at $d<24$ layers. The steep increase of phonon frequency in the few-layer thickness is observed in other layered compounds such as topological insulator $\left(\mathrm{Bi}_{2} \mathrm{Se}_{3}\right) 24$ and transition metal dichalcogenide $\left(\mathrm{MoS}_{2}, \mathrm{MoTe}_{2}\right)$ [25, 26] suggesting that it is possibly an universal behavior of layered $2 \mathrm{D}$ materials. The strong shift of phonon frequency shows that one can use $\omega_{0}$ to identify thickness of h-BN film as an alternative to
AFM or TEM.

We discuss briefly about the phonon frequency of our samples. For HT h-BN film the phonon frequency agrees with that of the single crystal for both $A_{2 \mathrm{u}}$ peak and $E_{1 \mathrm{u}}$ peak. However, for LT h-BN film, the $A_{2 \mathrm{u}}\left(E_{1 \mathrm{u}}\right)$ phonon frequency is notable lower (higher) than HT h-BN. When the substrate temperature is lower than the optimal temperature during the growth $\left(\mathrm{HT}=1400{ }^{\circ} \mathrm{C}\right.$ for our case) the $\mathrm{B}$ and $\mathrm{N}$ atoms cannot crystallize into fully relaxed, equilibrium lattice due to insufficient migration energy, causing some strain or tension in the film. We propose that the lattice strain may be one possible reason for the phonon frequency shifts. Also LT h-BN film may contain atomic vacancy and defects, which may also cause the phonon shifts.

\section{B. Graphene on h-BN}

To study the role of h-BN thin film as substrate for large scale graphene we transfer monolayer CVD graphene sheet on three different substrates, HT h-BN(2.5 nm thick $) / \mathrm{SiO}_{2} / \mathrm{Si}$, LT h$\mathrm{BN}(40 \mathrm{~nm}$ thick $) / \mathrm{SiO}_{2} / \mathrm{Si}$, and bare $\mathrm{SiO}_{2} / \mathrm{Si}$. Figure 4(a) shows the infrared transmission $T / T_{0}$ and reflection $R / R_{0}$ measured for the graphene $(\mathrm{G}) /$ substrate, where again the spectra $T$ and $R$ are normalized by the substrate $T_{0}$ and $R_{0}$. We extracted optical conductivity of graphene alone by applying the Kramers-Kronig constrained variational analysis to $T / T_{0}$ and $R / R_{0} \cdot[21$ ]

Figure 4(b) shows optical conductivity $\sigma_{1}$ of graphene. $\sigma_{1}$ represents interband transition $\sigma_{1}=\sigma_{0}\left(=\pi e^{2} / 2 h\right)$ of the Dirac carrier. 27, 28] At low $\omega, \sigma_{1}$ is suppressed because the transition becomes Pauli-forbidden. 29] The $\sigma_{1}$ curve shifts to lower energy as substrate is changed in the order $\mathrm{SiO}_{2} \rightarrow \mathrm{LT}$ h-BN $\rightarrow \mathrm{HT}$ h-BN. Theoretically the interband optical transition is expressed as

$$
\sigma_{1}=\frac{\sigma_{0}}{2}\left[\tanh \left(\frac{\hbar \omega+2 E_{\mathrm{F}}}{4 k_{\mathrm{B}} T}\right)+\tanh \left(\frac{\hbar \omega-2 E_{\mathrm{F}}}{4 k_{\mathrm{B}} T}\right)\right]
$$

where $E_{\mathrm{F}}$ and $T(=$ Temp) stand for the Fermi energy and sample temperature respectively. [30] We fit $\sigma_{1}$ using Eq.(1). The fitting parameters are summarized in Table I. For graphene on $\mathrm{SiO}_{2}$, we have $E_{\mathrm{F}}=214 \mathrm{meV}$ from the fit which, from the relation $E_{\mathrm{F}}=\hbar v_{\mathrm{F}} \sqrt{\pi n}$ and $v_{\mathrm{F}}=$ $1.1 \times 10^{8} \mathrm{~cm} / \mathrm{s}$, shows that Dirac band is doped by carrier, known to be hole, with density $n=2.9 \times 10^{12} \mathrm{~cm}^{-2}$.

TABLE I. Dirac carrier parameters measured from the interband transition conductivity $\sigma_{1}$ of graphene on different substrates. $E_{\mathrm{F}}=$ Fermi energy, $n=$ carrier density, and Temp* $=$ effective temperature.

\begin{tabular}{cccc}
\hline \hline Substrate & $E_{\mathrm{F}}(\mathrm{meV})$ & $n\left(10^{12} \mathrm{~cm}^{-2}\right)$ & Temp $^{*}(\mathrm{~K})$ \\
\hline HT h-BN $(2.5 \mathrm{~nm})$ & 93 & 0.5 & 300 \\
LT h-BN $(40 \mathrm{~nm})$ & 155 & 1.5 & 370 \\
$\mathrm{SiO}_{2}$ & 214 & 2.9 & 400 \\
\hline \hline
\end{tabular}



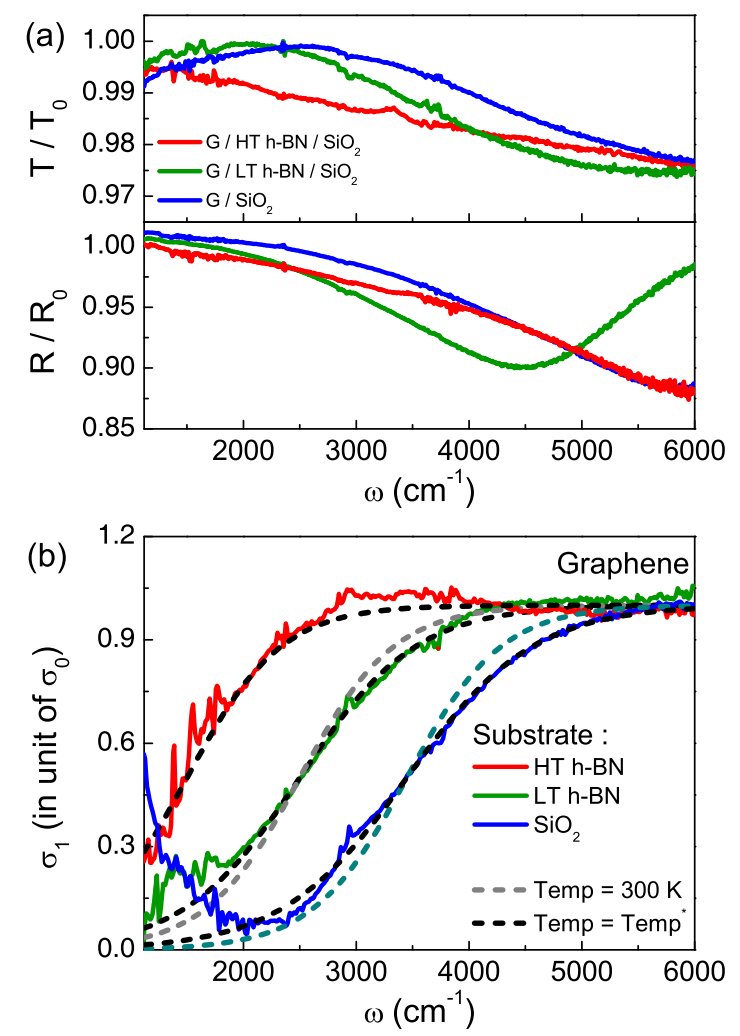

FIG. 4. (a) Transmission and reflection of graphene deposited on substrate, G/substrate, for three different substrates, HT h-BN/SiO 2 , LT h-BN/SiO 2 , and $\mathrm{SiO}_{2}$. (b) Optical conductivity $\sigma_{1}$ of graphene calculated from $T / T_{0}$ and $R / R_{0}$. Theoretical fits (see text) are shown by dashed curves.

The hole-doping of graphene is caused by various defect of $\mathrm{SiO}_{2}$ such as oxygen vacancy, surface dangle bonds, and others. For HT h-BN, we have significantly less values $E_{\mathrm{F}}=93 \mathrm{meV}$ and $n=5 \times 10^{11} \mathrm{~cm}^{-2}$. The latter is only $17 \%$ of that for $\mathrm{G} / \mathrm{SiO}_{2}$, showing that residual carrier has strongly decreased. It is interesting to compare the large scale CVD G/h-BN with single crystal G/h-BN. For exfoliated graphene on exfoliated h-BN, transport measurements showed $n$ is as small as $1.5 \times 10^{11} \mathrm{~cm}^{-2}$ at room- $T$ which is $10 \%$ of $n$ for single crystal G/SiO and $5 \%$ of $n$ for CVD G/SiO 2.7$]$ To gain better idea on the $n$ 's, we convert $n$ to the familiar transport $I$ $V$ curve of graphene. In the $I-V$ curve charge neutral voltage $V_{\mathrm{CNP}}$ is determined by $n$ as $n=\left(\epsilon_{\mathrm{r}} \epsilon_{0} / e d\right) V_{\mathrm{CNP}}$ where $\epsilon_{\mathrm{r}}=3.9$ and $d=300 \mathrm{~nm}$ are dielectric constant and thickness of $\mathrm{SiO}_{2}$, respectively. For G/ $\mathrm{SiO}_{2}$ sample we have $V_{\mathrm{CNP}}=40 \mathrm{~V}$. It is reduced to $V_{\mathrm{CNP}}=7 \mathrm{~V}$ for $\mathrm{G} / \mathrm{HT}$ h-BN and $V_{\mathrm{CNP}}=2 \mathrm{~V}$ for the single crystal G/h-BN. This result shows that large scale graphene was brought closer to the graphene on single crystal hBN by use of the CVD h-BN, and in fact is not far from $V_{\mathrm{CNP}}=0 \mathrm{~V}$, the ideal free standing state. For the G/LT h-BN, we have $E_{\mathrm{F}}=155 \mathrm{meV}$ and $n=1.5 \times 10^{12} \mathrm{~cm}^{-2}$. Those values lie between $\mathrm{SiO}_{2}$ and $\mathrm{HT}$ h-BN showing that LT h-BN is not as efficient as HT h-BN although it does reduce the residual carrier to some extent. High quality h-BN with well aligned BN planes is essential to achieve residual carrier-free graphene.

For Temp $=0$, Eq.(1) predicts that $\sigma_{1}$ changes suddenly from $\sigma_{1}=0$ to $\sigma_{1}=\sigma_{0}$ at $\hbar \omega=2 E_{\mathrm{F}}$. At elevated temperature (Temp $>0$ ), the carrier is thermally populated in the Dirac band and the sharp transition of $\sigma_{1}$ becomes broadened. For G/HT h-BN, $\sigma_{1}$ data agrees well with Eq.(1) calculated with the measurement temperature Temp $=300 \mathrm{~K}$. However for G/LT h-BN and G/SiO the $\sigma_{1}$ transition is broader than the theory curve. Here $\sigma_{1}$ can be fit only using higher Temp, Temp $=370 \mathrm{~K}$ for LT h-BN and Temp $=400 \mathrm{~K}$ for $\mathrm{SiO}_{2}$ as shown by black dashed curve. We define for convenience those Temp as effective temperature Temp*. Temp* $>300 \mathrm{~K}$ implies that there are extra source other than temperature that broadens the curve. Possibly the carrier is populated or redistributed in the Dirac band due to the defectdriven perturbation from the substrate, LT h-BN and $\mathrm{SiO}_{2}$. This postulation is supported by that Temp* is correlated with $E_{\mathrm{F}}$, i.e, Temp* decreases along with the residual carrier reduction as the substrate quality is increased as $\mathrm{SiO}_{2} \rightarrow$ LT h-BN $\rightarrow$ HT h-BN.

\section{CONCLUSION}

In conclusion we performed infrared measurement on large scale CVD h-BN thin film and CVD graphene/hBN heterostructure. For CVD h-BN sample, HT $\left(\right.$ Temp $\left.=1400{ }^{\circ} \mathrm{C}\right)$-grown thin film shows only $E_{1 \mathrm{u}}$ optical phonon like single crystal h-BN flake, demonstrating that $2 \mathrm{D}$ h-BN planes are highly aligned over the entire area of the film. On the other hand, LT $\left(1000{ }^{\circ} \mathrm{C}\right)$ grown film shows two phonon peaks, $E_{1 \mathrm{u}}$ and $A_{2 \mathrm{u}}$, due to tilting of the h-BN plane by $\theta=20 \sim 40^{\circ}$. For $\mathrm{G} / \mathrm{HT}$ h-BN samples, interband transition spectrum $\sigma_{1}$ shows that residual carrier density of graphene is strongly suppressed to $n=5 \times 10^{11} \mathrm{~cm}^{-2}$, from $n=2.9 \times 10^{12} \mathrm{~cm}^{-2}$ of $\mathrm{G} / \mathrm{SiO}_{2}$ and becomes comparable to $n$ of single crystal graphene on single crystal h-BN. Also the interband transition width of $\sigma_{1}$ is reduced from Temp $* 400 \mathrm{~K}$ for $\mathrm{G} / \mathrm{SiO}_{2}$ to the theoretical value Temp* $=300 \mathrm{~K}$ by using the HT h-BN film as substrate. The behaviors of $n$ and Temp* show that HT h-BN can decouple large scale graphene from $\mathrm{SiO}_{2}$ leading it closer to ideal, undoped Dirac semi-metallic state.

\section{ACKNOWLEDGMENTS}

This work was supported by Mid-career Researcher Program through National Research Foundation (NRF) grant funded by the MEST (NRF2014R1A2A2A01003448) and a grant from the Center for Advanced Soft Electronics under the Global Frontier Research Program through the NRF funded by the Ministry of Science, ICT and Future Planning (2011-0031630). 
[1] K. Watanabe, T. Taniguchi, and H. Kanda, Nat. Mater. 3, 404 (2004).

[2] A. K. Geim and I. V. Grigorieva, Nature 499, 419 (2013).

[3] E. Sichel, R. Miller, M. Abrahams, and C. Buiocchi, Phys. Rev. B 13, 4607 (1976).

[4] K. Watanabe, T. Taniguchi, T. Niiyama, K. Miya, and M. Taniguchi, Nat. Photonics 3, 591 (2009).

[5] T. Sugino and T. Tai, Jpn. J. Appl. Phys., Part 2 39, L1101 (2000).

[6] I. Jo, M. T. Pettes, J. Kim, K. Watanabe, T. Taniguchi, Z. Yao, and L. Shi, Nano Lett. 13, 550 (2013).

[7] C. R. Dean, A. F. Young, I. Meric, C. Lee, L. Wang, S. Sorgenfrei, K. Watanabe, T. Taniguchi, P. Kim, K. Shepard, et al., Nat. Nanotechnol. 5, 722 (2010).

[8] W. Gannett, W. Regan, K. Watanabe, T. Taniguchi, M. Crommie, and A. Zettl, Appl. Phys. Lett. 98, 242105 (2011).

[9] E. Kim, T. Yu, E. S. Song, and B. Yu, Appl. Phys. Lett. 98, 262103 (2011).

[10] G.-H. Lee, Y.-J. Yu, C. Lee, C. Dean, K. L. Shepard, P. Kim, and J. Hone, Appl. Phys. Lett. 99, 243114 (2011).

[11] J. Xue, J. Sanchez-Yamagishi, D. Bulmash, P. Jacquod, A. Deshpande, K. Watanabe, T. Taniguchi, P. JarilloHerrero, and B. J. LeRoy, Nat. Mater. 10, 282 (2011).

[12] K. K. Kim, A. Hsu, X. Jia, S. M. Kim, Y. Shi, M. Hofmann, D. Nezich, J. F. Rodriguez-Nieva, M. Dresselhaus, T. Palacios, et al., Nano Lett. 12, 161 (2011).

[13] Y. Gao, W. Ren, T. Ma, Z. Liu, Y. Zhang, W.-B. Liu, L.-P. Ma, X. Ma, and H.-M. Cheng, ACS nano 7, 5199 (2013).

[14] G. Kim, A.-R. Jang, H. Y. Jeong, Z. Lee, D. J. Kang, and H. S. Shin, Nano Lett. 13, 1834 (2013).

[15] J.-H. Park, J. C. Park, S. J. Yun, H. Kim, D. H. Luong, S. M. Kim, S. H. Choi, W. Yang, J. Kong, K. K. Kim, et al., ACS nano 8, 8520 (2014).

[16] A. Ismach, H. Chou, D. A. Ferrer, Y. Wu, S. McDonnell, H. C. Floresca, A. Covacevich, C. Pope, R. Piner, M. J. Kim, et al., ACS nano 6, 6378 (2012).
[17] J. W. Suk, A. Kitt, C. W. Magnuson, Y. Hao, S. Ahmed, J. An, A. K. Swan, B. B. Goldberg, and R. S. Ruoff, ACS Nano 5, 6916 (2011).

[18] A.-R. Jang, S. Hong, C. Hyun, S. I. Yoon, G. Kim, H. Y. Jeong, T. J. Shin, S. O. Park, K. Wong, S. K. Kwak, N. Park, K. Yu, E. Choi, A. Mishchenko, F. Withers, K. S. Novoselov, H. Lim, and H. S. Shin, Nano Lett. (2016).

[19] X. Li, W. Cai, J. An, S. Kim, J. Nah, D. Yang, R. Piner, A. Velamakanni, I. Jung, E. Tutuc, et al., Science 324, 1312 (2009).

[20] S. Bae, H. Kim, Y. Lee, X. Xu, J.-S. Park, Y. Zheng, J. Balakrishnan, T. Lei, H. R. Kim, Y. I. Song, et al., Nat. Nanotechnol. 5, 574 (2010).

[21] A. Kuzmenko, Rev. Sci. Instrum. 76, 083108 (2005).

[22] R. Geick, C. Perry, and G. Rupprecht, Phys. Rev. 146, 543 (1966).

[23] D. Hoffman, G. Doll, and P. Eklund, Phys. Rev. B 30, 6051 (1984).

[24] R. V. Aguilar, A. Stier, W. Liu, L. Bilbro, D. George, N. Bansal, L. Wu, J. Cerne, A. Markelz, S. Oh, et al., Phys. Rev. Lett. 108, 087403 (2012).

[25] C. Lee, H. Yan, L. E. Brus, T. F. Heinz, J. Hone, and S. Ryu, ACS Nano 4, 2695 (2010).

[26] M. Yamamoto, S. T. Wang, M. Ni, Y.-F. Lin, S.-L. Li, S. Aikawa, W.-B. Jian, K. Ueno, K. Wakabayashi, and K. Tsukagoshi, ACS Nano 8, 3895 (2014).

[27] V. Kravets, A. Grigorenko, R. Nair, P. Blake, S. Anissimova, K. Novoselov, and A. Geim, Phys. Rev. B 81, 155413 (2010).

[28] D.-H. Chae, T. Utikal, S. Weisenburger, H. Giessen, K. v. Klitzing, M. Lippitz, and J. Smet, Nano Lett. 11, 1379 (2011).

[29] Z. Li, E. A. Henriksen, Z. Jiang, Z. Hao, M. C. Martin, P. Kim, H. Stormer, and D. N. Basov, Nat. Phys. 4, 532 (2008).

[30] K. F. Mak, M. Y. Sfeir, Y. Wu, C. H. Lui, J. A. Misewich, and T. F. Heinz, Phys. Rev. Lett. 101, 196405 (2008). 